\section{La inclusión de la extensión en la formación de los estudiantes de la Universidad Nacional del Litoral}

En la UNL, el proceso de institucionalización de incorporación curricular comenzó en el año 2007, a partir de una propuesta elevada por los estudiantes al Consejo Superior de la Universidad, con el fin de "instaurar condiciones institucionales que favorezcan la incorporación de las prácticas de extensión a las propuestas curriculares de las carreras a manera de profundizar alternativas de formación de profesionales sólidos y fundamentalmente comprometidos con la sociedad" (Res. HCS 274/07).

Con la creación del Área de Incorporación Curricular en el marco de la Secretaría de Extensión en el año 2008, se inició un exhaustivo análisis sobre las prácticas de extensión en las Unidades Académicas a fin de identificar aportes sustanciales basados en las experiencias de alumnos y docentes. Este análisis contribuyó a esbozar un marco teórico para delinear los aspectos más relevantes que caracterizan a las prácticas de extensión, y a definir — desde una perspectiva pedagógica - cómo éstas pueden ser pensadas como estrategias privilegiadas en la construcción del conocimiento. En ese documento preliminar se enfocó a la extensión desde múltiples dimensiones: en su "dimensión social", en su "dimensión dialógica" y en su "dimensión pedagógica".

Siguiendo con el trabajo, se desarrollaron talleres con los secretarios académicos y de extensión, directores de carrera y de departamento de las diferentes carreras de grado, con el objeto de plantear estrategias para la implementación de prácticas de extensión en el currículum. Paralelamente, se organizó y desarrolló un Curso de Extensión destinado a docentes denominado "La resolución de problemas abiertos como estrategia de enseñanza en la Universidad. El trabajo en el área de extensión" y un Curso de Posgrado "Las prácticas académicas en espacios socio culturales, productivos y/o comunitarios. Pensando la educación experiencial”, ambos a cargo de la profesora Alicia Camilloni.

Avanzando con el proceso, se planificó un doble relevamiento. Por un lado, se buscó identificar la percepción de diferentes actores universitarios con relación a la extensión y a la propuesta específica de su incorporación en los currículos. Desde allí se buscaba conocer tanto el interés que tienen los docentes respecto de la generación y desarrollo de actividades de extensión y su integración con la docencia y la investigación, como de la valoración y el interés en participar que los actores universitarios demuestran para llevar adelante actividades de este tipo.

El segundo nivel del relevamiento consistió en identificar posibles espacios curriculares a partir de los cuales se podrían realizar prácticas de extensión. Para ello fueron consultados los Secretarios Académicos y de Extensión y Directores de Carrera y de Departamentos, y se realizó un análisis de los planes de estudios de todas las carreras de grado de la UNL.

Una vez sistematizada toda la información, se trabajó junto a la Secretaría Académica de la UNL en torno a la implementación y acreditación de dichas prácticas por parte de los estudiantes, y se logró acordar una propuesta de resolución y reglamento sobre la incorporación curricular de las prácticas de extensión en las carreras de grado de la UNL. 
+E entrevista por Cecilia lucci

\section{Alicia W. de Camilloni}

Especialista en didáctica, currículo y educación superior. Profesora Emérita e Investigadora de la Universidad de Buenos Aires. Dicta cursos de posgrado en distintas universidades. Asesora de un gran número de organismos educativos nacionales e internacionales. Publicó numerosos trabajos en revistas especializadas y es coautora de varios libros. En la Universidad Nacional del Litoral, es docente en maestrías y posgrados y es asesora del Área de Incorporación Curricular de la Secretaría de Extensión.

\section{- ¿Qué desafíos supone para la Universidad pública la definición de una política que apueste a integrar las misiones de docencia, investigación y extensión?}

- En principio, hay que decir que esta es una época particularmente desafiante para las universidades porque los retos a los que hoy deben responder son de naturaleza heterogénea y de magnitud muy significativa. Se trata de cambios rápidos, variados y simultáneos que afectan a las universidades en aspectos estructurales, académicos, administrativos y culturales. Estos cambios se traducen en un numeroso conjunto de problemas interrelacionados que exigen adaptabilidad para responder a las demandas variadas de un contexto cambiante, sin perder los principios de la moral académica y para servir mejor al interés común.

Es interesante advertir que la educación universitaria dejó de ser un proyecto individual para convertirse en un instrumento de la sociedad para su propia transformación.

En tanto integrante de la sociedad civil y del estado, la universidad pública constituye un factor estratégico para promover el crecimiento económico, social y cultural y, por consiguiente, el bienestar de la población. De allí la responsabilidad social que tiene hacia los individuos, grupos y organizaciones que se movilizan en demanda de la satisfacción de necesidades y que, además, como institución generadora de personas competentes en la producción de bienes y servicios, puede contribuir a responder a esos requerimientos.

En el marco actual de la vida institucional, en el que la multiplicidad de misiones y funciones se impone en la universidad, no cabe duda de que, con mayor intensidad que en el pasado, las misiones de docencia, investigación y extensión deben estar entretejidas. La investigación, en tanto permite reconocer, diagnosticar e identificar las causas de los problemas sociales y programar con eficiencia sus mejores soluciones. La docencia debe formar a los estudiantes y graduados que estén en condiciones de efectuar una efectiva intervención en el campo social profesional o no profesional y facilitar la relación teoría-praxisteoría como dispositivo de formación. En ambos casos, con el fin de realizar las acciones sociales directas que contribuyan a la solución de los problemas sociales e individuales.

Sin embargo, cabe señalar que el colocar a la universidad al servicio de la sociedad plantea numerosos interrogantes respecto de cómo se define a la sociedad, qué lazos deben unirlas y cuáles son, si deben establecerse, los valores predominantes que rijan esa relación. Más aun: cuáles han de ser las voces que deben ser escuchadas; cómo procesa la universidad —que en su interior también es heterogénea-, la multidiversidad de la sociedad con la que se encuentra en diálogo; cuáles han de ser los actores representativos de la definición de las necesidades y de los proyectos sociales; qué necesidades deben atenderse y cuáles no; quién determina cuáles han de ser las prioridades presentes y futuras que la universidad deberá atender; cuáles son los canales que se abren para que, en la escucha de la comunidad, la universidad respetuosamente aprenda de ella y se nutra de su riqueza cultural. 
- Cuándo Ud. habla de incorporación de la extensión en la formación de los estudiantes recurre a dos conceptos que son el de educación experiencial y aprendizaje-servicio. Brevemente ¿qué significan esos conceptos?
- ¿Qué esfuerzos institucionales requiere su implementación?
- La educación experiencial es una estrategia de enseñanza con enfoque holístico, que está destinada a relacionar el aprendizaje académico con la vida real. Con ese fin, propone al alumno realizar actividades en las que, a partir de esa conexión con la práctica, ponga a prueba, en situación auténtica, es decir, de la vida real, las habilidades y los conocimientos teóricos que posee, evalúe sus consecuencias, enriquezca esos conocimientos y habilidades e identifique nuevos problemas. Cuando el aprendizaje es experiencial, quien aprende lo hace en las condiciones sociales de la utilización auténtica de los conocimientos. Aprende en un marco en que se apunta al logro simultáneo de fines comunitarios y fines educativos. Aprende a usar los conocimientos en el trabajo. Pero para que se sostengan los valores a los que antes nos referimos, debe tratarse de un trabajo con organización democrática, que otorgue a la vez derechos y responsabilidad al estudiante, en el que se promueva la adopción de decisiones autónomas y que debería estar lejos de las organizaciones que Lewis Coser denominaba "organizaciones glotonas" (greedy institutions) que exigen lealtad total a los individuos, ya que ellas reclamarían acciones no autónomas, contrarias al tipo de formación que la universidad debe promover en sus estudiantes. En la realización de esta actividad formativa, el estudiante requiere orientación, de tal modo que se salvaguarde su libertad y se estimule a un tiempo su creatividad. Para que un programa de educación experiencial, en sus variadas formas pueda configurarse de

- Se pueden enumerar varios aspectos, pero en primer término, implica manifestar claramente el compromiso formal de toda la universidad. Este compromiso supone reconocer la contribución que las organizaciones "socias" hacen a la universidad $y$, a la vez, aceptar que los estudiantes pueden aportar respuestas a problemas que se presentan en la realidad.

La integración de la extensión en el currículum implica, entre otras cuestiones, desarrollar un plan estratégico, que sea consensuado con las organizaciones que intervienen, en el que puedan tener participación los alumnos y en el que se fijen claramente metas mediatas e inmediatas. Supone un esfuerzo de articulación manera adecuada la universidad, debe crear condiciones que permita una rápida toma de decisiones en cuanto a la apertura y cierre de actividades, es preciso que establezca redes de relaciones con instituciones "socias", que facilite la rápida adaptación de tutores y estudiantes. Para ello, debe contar con rasgos significativos de permeabilidad a las necesidades, a los problemas y a las demandas de la comunidad, escuchando y haciendo sus propias lecturas. David Kolb es un referente fundamental para comprender los alcances del concepto de educación experiencial. Entre las diversas modalidades de la educación experiencial, se encuentra el aprendizaje-servicio que es una estrategia de enseñanza en la que los estudiantes aplican sus habilidades y conocimientos académicos y profesionales específicos para satisfacer necesidades reales en respuesta a requerimientos explícitos de la comunidad. Además de servir a la formación profesional, el aprendizaje-servicio contribuye a la educación para la ciudadanía y la formación ética de los estudiantes. Les facilita, también, el conocimiento del contexto comunitario y social al tiempo que les permite brindar servicios de valor positivo en respuesta a demandas de satisfacción de necesidades externas o internas a la universidad. El aprendizaje-servicio pone el acento en los dos términos que constituyen esta expresión, el aprendizaje y el servicio, y no sólo en uno de ellos. Se diferencia, así, de la práctica profesional que se centra en el aprendizaje y del voluntariado que lo hace en el servicio.

entre diversas asignaturas o con otras funciones de la universidad porque los problemas con que se encuentra el estudiante son siempre complejos y no podrán ser abordados desde una única perspectiva disciplinaria, y ni siquiera, con frecuencia, monoprofesional. Significa contar con una organización de coordinación eficiente, en la que haya una buena circulación de información entre la universidad y los socios, entre los docentes y los estudiantes. Necesita, sin lugar a dudas, el compromiso de un número importante de docentes que tengan una formación adecuada para poder ejercer las funciones tutoriales a fin de resguardar el trabajo del estudiante y garantizar la calidad del servicio que se presta. 


\section{- La implementación de esta política en el marco de los espacios curriculares ¿qué impacto supone que traería aparejado sobre el propio campo de la extensión universitaria, desde sus más diversas definiciones?}

- Las acciones de extensión se vienen realizando desde hace mucho tiempo en las universidades públicas. Son ejemplos de esto: los servicios de salud, los hospitales universitarios, los trabajos de divulgación científica, las acciones de desarrollo y transferencia que se hacen por convenios con organismos estatales, con ONGs o con empresas. Sin embargo, lo que sucede con estas acciones es que el papel que juegan los estudiantes es de alguna manera aleatorio. Muchas veces se trata de trabajos de cátedras que están a cargo de un grupo de docentes y que desarrollan acciones en función de algún tipo de demanda que recibieron o, incluso, en virtud de un convenio de venta de servicios. Incorporar la extensión en el currículo, en cambio, busca rescatar el papel de formación que tiene la extensión para los estudiantes al ponerlos en contacto con la realidad.

Para ello el estudiante deberá realizar una tarea en común con la comunidad — sin que ello implique hacer asistencialismo-, en el que ponga en juego su formación académica y su compromiso ciudadano. Se trata también de aprender a escuchar y a trabajar con miembros de la comunidad.

Hay carreras que desde hace varios años trabajan de esta manera, sin embargo, lo que falta es una definición política de la universidad en su conjunto para que todas las carreras asuman el desafío de incorporar la extensión en la formación sistemática de los alumnos.

Esta definición posiciona a la universidad ya no sólo como una institución que provee servicios sino como alguien que construye un marco común de trabajo e incluye a los estudiantes en esta prestación. Por eso se habla de socios, y no de receptores externos. Se habla de la generación de una sociedad entre la universidad y las comunidades con las que se va a trabajar. $Y$ es en este marco en el que la universidad aprende de esas comunidades.

El tema qué es o cómo debe definirse la extensión se viene discutiendo desde hace mucho tiempo. Sin embargo, cuando este tipo de prácticas se asume como política institucional, lo que se institucionaliza es una concepción de la extensión que venía siendo discutida teóricamente o sostenida desde los discursos, y que, muchas veces, ha resultado poco actuada. En la medida en que se asuma, efectivamente, con un fuerte compromiso institucional, la cuestión de la extensión no se limitará a su redefinición teórica sino que se planteará en términos de acción. $A$ mi juicio, esa es una de las maneras de rescatar, realmente, a la extensión como instrumento de política universitaria. 